\title{
A simple instrument for assessing stress in clinical practice
}

\author{
Nicoletta Sonino, Giovanni A Fava
}

\begin{abstract}
Summary
Methods to assess the role of stress factors in patients with medical conditions are often rather complex, require specific training, and are difficult to use in clinical practice. We attempted to develop a short index tailored to a busy clinical setting, which would be easy to use while providing adequate individual information. This index (Psychosocial Index) was largely derived from well-established instruments, such as Kellner's Screening List for Psychosocial Problems. In addition, on the basis of the patient's self-report of items, the clinician is asked to rate four dimensions of the patient's life: stress, wellbeing, psychological distress, and illness behaviour. The questionnaires of 34 female patients with functional medical disorders were first rated by an internist and afterwards, blindly, by a psychiatrist. Agreement between the two raters was excellent, as measured by the intraclass correlation coefficient. It is hoped that this Psychosocial Index may provide a new tool for psychosomatic research and practice.
\end{abstract}

Keywords: stress; rating scales; quality of life; functional medical disorders

There is growing awareness of the need of a quick assessment of psychosocial variables in clinical practice. Somatisation - the tendency to experience and communicate psychological distress in the form of physical symptoms and to seek medical help for them ${ }^{1}-$ is a widespread clinical phenomenon. Particularly when symptoms lack an adequate physical explanation, even after a reasonable work-up, the physician must evaluate the contribution of life stress. One of the most widely cited definitions of stress was provided by Lazarus and Folkman ${ }^{2}$ : "Psychological stress is a particular relationship between the person and the environment that is appraised by the person as taxing or exceeding his or her resources and endangering his or her well-being" (p 19). Two central features of this definition are its interpersonal emphasis and the importance it places on assessment of events. ${ }^{3} \mathrm{~A}$ need for the integration of objective and subjective aspects has emerged in conceptual and methodological issues such as differentiation of major and daily stresses, ${ }^{3}$ subjective appraisal, ${ }^{4}$ and the interactional role of affective disturbances. $^{5}$ The most valid and reliable information in life-events research has been achieved by interview methods which permit enough probing to establish detail. ${ }^{6}$ These methods are often rather complex, require specific training and are difficult to use in clinical practice. Similar considerations apply to the detection of psychological distress. Studies have consistently shown that primary care physicians fail to diagnose and/or appropriately refer at least $50 \%$ of patients suffering from common mental disorders, such as anxiety and depression. ${ }^{7}$ Structured diagnostic interviews are again the most suitable instruments ${ }^{8}$ but, even in their most abridged and primary care oriented forms, ${ }^{7}$ they take considerable time. Self-rating questionnaires and use of cut-off scores are another viable option, ${ }^{8}$ but require scoring and involve delay in feedback to responders. ${ }^{9}$ This is a crucial issue, not least for its clarification opportunities.

Kellner $^{10}$ developed the Screening List for Psychosocial Problems (SLP), a self-rating scale of problems and symptoms with 118 questions, for clinical work in psychiatry. The SLP was extensively validated ${ }^{10}$; it was found to discriminate between different populations (also in its Italian version ${ }^{11}$ ) and to be sensitive to change.

The domain of clinimetrics is concerned with quantitative methods in the collection and analysis of clinical phenomena, such as types, severity and sequence of symptoms, problems of functional capacity, and reasons for medical decisions, with emphasis on clinical judgement. ${ }^{12}$ There are important differences between clinimetric and psychometric principles. ${ }^{8}{ }^{12}$ Homogeneity of items on a single scale is a crucial characteristic in psychometrics: however, the same properties that give an index a high score for homogeneity (redundance), also obscure its ability to detect an altered state, ${ }^{12}$ and are thus regarded as undesirable in clinimetrics.

We set out to develop a rating scale, based on clinimetric principles, that is simple to use in a busy clinical setting (being based on a relatively short, self-rated, questionnaire), which could be integrated with clinical judgement (by observer-rated methods), and which would provide a first-line, comprehensive, assessment of psychosocial features.

\section{Methods}

INSTRUMENT

The rating scale, called the Psychosocial Index (PSI), consists of a self-rated (box 1) and an observer-rated (box 2) part. The self-rated part 


\section{Self-rated items of the psychosocial index}

NAME ................................................... DATE

1 Date of birth: Day ................... Month ................... Year ...

2 Sex: Male / Female

3 Occupation

Occupation of spouse

4 Marital Status: Single / Married / Divorced / Separated / Widowed

5 Have you ever been hospitalized? Yes / No

6 Please list illnesses, surgical operations and other treatments and give dates

7 Are you allergic to any drugs or substances?

8 What medications are you taking at present?

9 Do you drink alcohol? Yes / No

10 Do you smoke? Yes / No

11 Do you take recreational drugs? Yes / No

12 Do you drink coffee or tea? Yes / No

Did any of the following happen to you in the past year? (Yes/No)

13 Death of a family member or a very close friend

14 Separation from spouse or long-time partner

15 Recent change of job

16 Moving within the same city

17 Moving to another city

18 Financial difficulties

19 Legal problems

20 Beginning of a new relationship

21 How many hours do you work per week? .

Please answer the following questions (Yes/No)

22 Are you satisfied with your work?

23 Do you feel under pressure at work?

24 Do you get along with your colleagues at work?

25 Do you get along with your spouse or partner?

26 Do you get along with other relatives?

27 Has any close relative been seriously ill in the past year?

28 Do you feel tension at home?

29 Do you live by yourself?

30 Do you feel lonely?

31 Do you have anyone whom you can trust and confide in?

32 Do you get along well with people?

33 Do you often feel overwhelmed by the demands of every day life?

34 Do you often feel you cannot make it?

35 Do you tend to be influenced by people with strong opinions?

36 Do you tend to worry about what other people think of you?

Please describe any problems or difficulties you have had recently

and indicate how much they have troubled you by marking the

appropriate column (Not at all / Only a little / Somewhat / A great deal)

37 It takes a long time to fall asleep

38 Restless sleep

39 Waking too early and not being able to fall asleep again

40 Feeling tired on waking up

41 Stomach, bowel pains

42 Heart beating quickly or strongly without a reason

43 Feeling dizzy or faint

44 Feelings of pressure or tightness in head or body

45 Breathing difficulties or feeling of not having enough air

46 Feeling tired or a lack of energy

47 Irritable

48 Sad or depressed

49 Feeling tense or 'wound up'

50 Lost interest in most things

51 Attacks of panic

52 Do you believe that you have a physical disease but that doctors have not diagnosed it correctly?

53 When you read or hear about an illness, do you get similar symptoms?

54 When you notice a sensation in your body, do you find it difficult to think of something else?

55 How do you rate the quality of your life? (Excellent / Good / Fair / Poor / Awful)

\section{Box 1}

included in the SLP in order to provide an appraisal of daily, work, and interpersonal stress. Questions 31-36 were derived from another well-validated instrument, Ryff's Scale of Psychological Well-being, an 84-item inventory that covers six areas of well-being: autonomy, environmental mastery, personal growth, positive relations with others, purpose in life and self-acceptance. ${ }^{14}$ Finally, a simple direct question on quality of life, following Gill and Feinstein's ${ }^{15}$ recommendation, was provided.

Some questions of the PSI involve specific responses (1-8 and 21 ), most require a yes/no answer $(9-20,22-36)$, while others are rated on a Likert 0-3 scale (from 'not at all' to 'a great deal') (37-54); one item (55) has five possible choices.

By scanning the patient's responses, the clinician is asked to rate four dimensions of the patient's life: stress, psychological distress, abnormal illness behaviour, and well-being (box 2). In addition to severity of symptoms, this rating may put a differential emphasis on items according to clinimetric principles. ${ }^{12}$ Stress is thus rated on the basis of the patient's responses to questions 13-30, well-being on responses to 31-36 and 55, psychological distress on responses to $37-51$, and abnormal illness behaviour on responses to $52-54$.

\section{DATA COLLECTION}

Thirty-four consecutive female medical outpatients with a functional medical disorder were evaluated by an internist (NS). All patients were studied at the Institute of Semeiotica Medica of Padova University. The mean age of the patients was $35.7(\mathrm{SD}=10.9)$ years. Their presenting complaints included cardiovascular symptoms, gastrointestinal disturbances, globus, menstrual abnormalities, mild hyperprolactinaemia, skin manifestations, fatigue, dizziness, and headache. After medical examination and work-up had indicated a functional disorder, all patients were asked to complete the questionnaire. The internist rated patients' responses. A psychiatrist (GAF), blind to the internist's rating, rated patients' responses based only on the questionnaires.

VALIDATION DESIGN AND STATISTICAL METHODS The PSI consists mainly of selected items from previously validated instruments; the novel part of the scale, which requires validation, is the observer-rated part. The inter-rater agreement (reliability) of the observer-rated part was therefore assessed using intraclass correlation coefficients to evaluate the agreement between the two raters. ${ }^{16}$ (Simple correlation coefficients are inadequate in these cases, since they indicate trends rather than concordance.)

\section{Results}

includes 55 items; 38 of which (questions 1-20 and 37-54) were derived from the 118 of the SLP, eliminating all sources of redundancy. Questions 21-30 were derived from Wheatley Stress Profile, ${ }^{13}$ another validated instrument. They were added to the list of life events
The intraclass correlation coefficients were 0.88 for rating stress, 0.94 for well-being, 0.89 for psychological distress, and 0.90 for illness behaviour. Since all intraclass correlation coefficients were above 0.80 , there was excellent inter-rater concordance. 


\begin{tabular}{|c|c|c|c|c|c|}
\hline \multicolumn{6}{|c|}{ Observer-rated scales of the psychosocial index } \\
\hline & $\begin{array}{l}\text { Highly stressful } \\
\text { life }\end{array}$ & & $\begin{array}{l}\text { Stressful } \\
\text { life }\end{array}$ & & $\begin{array}{l}\text { Non-stressful } \\
\text { life }\end{array}$ \\
\hline Stress & 5 & 4 & 3 & 2 & 1 \\
\hline Well-being & $\begin{array}{l}\text { Excellent } \\
5\end{array}$ & $\begin{array}{l}\text { Good } \\
4\end{array}$ & $\begin{array}{l}\text { Fair } \\
3\end{array}$ & $\begin{array}{l}\text { Poor } \\
2\end{array}$ & Absent \\
\hline & Incapacitating & Severe & Moderate & Slight & Absent \\
\hline Psychological distress & & 4 & 3 & & \\
\hline Abnormal illness behaviour & 5 & 4 & 3 & 2 & 1 \\
\hline
\end{tabular}

Box 2 and assessment. The psychosocial dimensions to be rated have considerable clinical importance. The rating of stress attempts an integration of both perceived and objective stress, life events and daily stress. ${ }^{1-7}$ Such evaluation may be linked to the individual's potential for coping and social support, subsumed here under the rating of well being. The underlying concept has considerable overlaps with that of quality of life, without, however, being flawed by problems of definition. ${ }^{15} 17$ Sleep disturbances, somatisation, anxiety, depression, and irritability are subsumed under the rubric of psychological distress. This rubric is of immediate practical value for an internist and allows further diagnostic refinement to be obtained through consultation-liaison psychiatry. Abnormal illness behaviour - the persistence of a maladaptive mode of perceiving, experiencing, evaluating and responding to one's health status, including hypochondriasis and bodily preoccupation $^{18}$ - encompasses another crucial aspect of somatisation and the patientdoctor relationship.

Even though the PSI generates self-rating scores, it is not intended to be used in such a way, but rather as a tool for identifying stress and distress in medical patients. It may allow clinicians to become aware of the degree of stress a patient is subjected to, providing a positive clue for a diagnosis of functional medical disturbance (otherwise identified mainly by excluding organic factors). It may provide preliminary grounds for specific questions as to psychological distress during medical examination, leading to diagnostic and therapeutic decisions or specialist referral. clinical judgement for the specific area of concern, not necessarily linked to the numerical score of self-reported items, with opportunities for clarification during the medical interview

1 Lipowski ZJ. Somatization. Psychother Psychosom 1987;47:160-7.

Lazarus RS, Folkman S. Stress, appraisal, and coping. New York: Springer; 1984.

Wagner BM. Major and daily stress and psychopathology. Stress Med 1990;6:217-66.

4 Peacock EJ, Wong PTP. The stress appraisal measure (SAM). Stress Med 1990;6:227-36.

Endler NS, Parker JDA. Stress and anxiety. Stress Med 1990; 6:243-8.

6 Paykel ES. Methodology of life events research. In: Fava GA, Wise TN, eds, Research paradigms in psychosomatic medicine. Basel: Karger, 1987; pp 13-29.

7 Spitzer RL, Williams JBW, Kroenke K, et al. Utility of a new procedure for diagnosing mental disorders in primary care. FAMA 1994;272:1749-56.

8 Fava GA. Screening and diagnosis of depression. Dis Manage Health Outcomes 1997;2:1-7.

9 Whitfield M. Giving feedback to questionnaire responders an essential task? Br $\mathcal{f}$ Gen Pract 1997;47:241-3.
This study was supported by grant 545/01/94 (Ricerca Sanitaria Finalizzata) from Regione Veneto (Venice, Italy) to Dr Sonino.
10 Kellner R. A problem list for clinical work. Ann Clin Psychiatry 1991;3:125-36.

11 Sonino N, Fava GA, Mani E, Belluardo P. Quality of life of hirsute women. Postgrad Med f 1993;69:186-9.

12 Feinstein AR. Clinimetrics. New Haven, CT: Yale University Press, 1987.

13 Wheatley D. The stress profile. Br f Psychiatry 1990;156: 658-88.

14 Ryff CD, Singer B. Psychological well-being. Psychother Psychosom 1996;65:14-23.

15 Gill TM, Feinstein AR. A clinical appraisal of the quality of quality-of-life measurements. $¥ A M A$ 1994;272:619-26.

16 Fleiss JL. The design and analysis of clinical experiments. New Fleiss JL. The design and an

17 Sonino N, Fava GA, Fallo F, Boscaro M. Psychological distress and quality of life in endocrine disease. Psychother Psychosom 1990;54:140-4.

18 Kellner R. Somatization and hypochondriasis. New York: Praeger, 1986. 